\title{
Steady Stokes flow between confocal semi-ellipses
}

\author{
I. V. Makeev, I. Yu. Popov \\ ITMO University, Kronverkskiy, 49, St. Petersburg, 197101, Russia \\ popov1955@gmail.com
}

PACS 68.90.+g, 05.60.-k, 62.10.+s

DOI 10.17586/2220-8054-2016-7-2-324-331

\begin{abstract}
Analytical solutions for the Stokes equations in a cavity bounded by two confocal semi-ellipses and two line segments are derived here. The exact solution for the stream function, in the form of a Fourier series, is obtained. Eddy structure is described for different boundary conditions.
\end{abstract}

Keywords: Stokes flow, biharmonic equation.

Received: 22 January 2016

\section{Introduction}

Micro- and nanoflows are new fields of nanotechnology. Flow through nanostructures is known to have many interesting unusual peculiarities [1]. Particularly, one observes a phenomenon analogous to superfluidity [2], the dependence of viscosity on the nanotube's diameter [3] and other such effects. The theory of nanoflow is not well-developed. There are only a few works suggesting theoretical explanations for these phenomena (see, e.g., [4-6]). It has been shown that hydrodynamic equations should be modified for nanoflows [7], but the Stokes approximation is appropriate due to the smallness of the Reynolds number [8].

The most interesting question is about the eddy structure for nanoflow. The information about it can be used for several nanophysical and nanochemical applications. Particularly, it was shown experimentally that there is a separation of a fluid's components in nanochannels [9]. Among other reasons, it is related with the existence of eddies. One observes component separation within an eddy due to differences in the components' densities. As a result, this property opens the way for the creation of a chemical nanoreactor. Namely, due to component separation, the reagents needed for some chemical reaction are collected within some local domain inside the eddy. Correspondingly, one has strong localization of the chemical reaction in this domain only. We have the opportunity to use this phenomenon, only if we can predict the eddy structure for different system parameters. Particularly, if the flow is induced by the boundary condition, we require information about the dependence of the eddy structure on the boundary conditions.

One can mention that similar processes take place in non-autonomous phases [10]. It is interesting to note that the analogous mathematical problem also present in geophysics [11].

Stokes flows in various domains were studied by analytical methods in many papers. There are a number of works describing the flow over a rectangular cavity [12-18]. The solutions for the Stokes equations for the annular cavities were investigated in [19-25]. Stokes flow in regions partially bounded by segments of ellipses was considered in [26,27]. Corner eddies in the Stokes flow problems were studied in [28-30].

In the present paper, we describe the Stokes flow in a horseshoe domain formed by two semi-ellipses and two segments (Fig. 1). The flow is induced by inhomogeneous boundary conditions. We investigate the eddy structure for different boundary conditions. Although the 
investigation was inspired by the nanoflows problem, the result concerns the classical Stokes flow problem.

\section{Problem formulation and solution}

Let us consider Stokes flow in a cavity bounded by two confocal semi-ellipses, $S_{1}$ and $S_{2}$ and two line segments, $A B$ and $C D$ (Fig. 1). Flow in the cavity is induced by the velocities $V_{\text {top }}$ and $V_{b o t}$ at $S_{1}$ and $S_{2}$ respectively. On segments $A B$ and $C D$, we assume free-slip conditions.

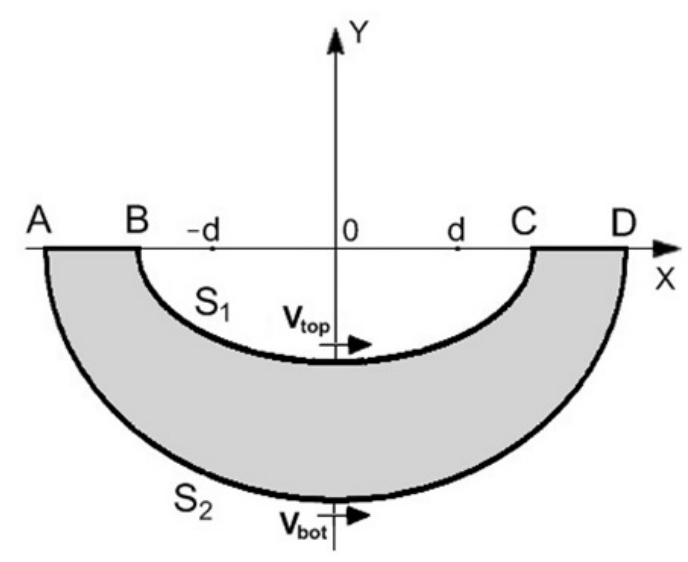

FIG. 1. The domain in the Cartesian coordinates. $(-d, 0),(d, 0)$ are the ellipse foci

In the $2 \mathrm{D}$ case, the Stokes equations can be reduced to the biharmonic equation for the stream function $\Psi$ :

$$
\Delta^{2} \Psi=0 .
$$

We make the transformation of the cavity to the elliptic coordinates system:

$$
x=d \cos \left(\xi_{2}\right) \cosh \left(\xi_{1}\right), \quad y=d \sin \left(\xi_{2}\right) \sinh \left(\xi_{1}\right) .
$$

The Laplace operator takes the form:

$$
\Delta=\frac{1}{d^{2}\left(\cosh ^{2}\left(\xi_{1}\right)-\cos ^{2}\left(\xi_{2}\right)\right)}\left(\frac{\partial^{2}}{\partial \xi_{1}^{2}}+\frac{\partial^{2}}{\partial \xi_{2}^{2}}\right) .
$$

Our domain transforms to a rectangle on Fig. 2. Semi-ellipses $S_{1}, S_{2}$ in elliptic coordinate system will convert to segments $\xi_{1}=\xi_{01}, \xi_{1}=\xi_{02}, \pi \leq \xi_{2} \leq 2 \pi$.

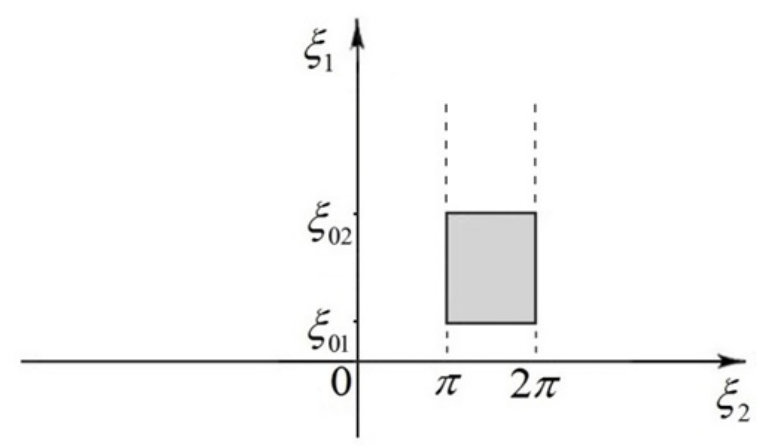

FIG. 2. The domain in elliptic coordinates 
Let us consider the following chain of equations:

$$
\begin{gathered}
\Delta \Psi=\Psi_{1}, \\
\Delta \Psi_{1}=0 .
\end{gathered}
$$

The boundary conditions for stream function are:

$$
\begin{gathered}
\Psi\left(\xi_{1}, 0\right)=0, \quad \Psi\left(\xi_{1}, \pi\right)=0, \quad \xi_{01} \leq \xi_{1} \leq \xi_{02} ; \\
\Psi\left(\xi_{01}, \xi_{2}\right)=0, \quad \Psi\left(\xi_{02}, \xi_{2}\right)=0, \quad \pi \leq \xi_{2} \leq 2 \pi ; \\
\left.\frac{\partial^{2} \Psi}{\partial \xi_{2}^{2}}\right|_{\xi_{2}=\pi}=0,\left.\quad \frac{\partial^{2} \Psi}{\partial \xi_{2}^{2}}\right|_{\xi_{2}=2 \pi}=0, \quad \xi_{01} \leq \xi_{1} \leq \xi_{02} ; \\
\frac{1}{h} \frac{\partial \Psi}{\partial \xi_{1}}\left(\xi_{01}, \xi_{2}\right)=V_{S_{1}}\left(\xi_{2}\right), \quad \frac{1}{h} \frac{\partial \Psi}{\partial \xi_{1}}\left(\xi_{02}, \xi_{2}\right)=V_{S_{2}}\left(\xi_{2}\right), \quad \pi \leq \xi_{2} \leq 2 \pi .
\end{gathered}
$$

Lame coefficients are: $h_{\xi_{1}}=h_{\xi_{2}}=h=d \sqrt{\cosh ^{2}\left(\xi_{1}\right)-\cos ^{2}\left(\xi_{2}\right)}$.

The function $\Psi_{1}\left(\xi_{1}, \xi_{2}\right)$ in (3) can be found by standard separation of variables (for calculations we take $m$ terms):

$$
\Psi_{1}=\sum_{k=1}^{m}\left(c_{1 k} e^{k \xi_{1}}+c_{2 k} e^{-k \xi_{1}}\right) \sin \left(k \xi_{2}\right) .
$$

We derive a solution of Eq. (2) in the form of the Fourier series:

$$
\begin{gathered}
\Psi\left(\xi_{1}, \xi_{2}\right)=\sum_{k=1}^{n} \Psi_{1 k}\left(\xi_{1}\right) \sin \left(k \xi_{2}\right), \quad \pi \leq \xi_{2} \leq 2 \pi \\
\Psi_{11}\left(\xi_{1}\right)=a_{31} e^{\xi_{1}}+a_{41} e^{-\xi_{1}}+\frac{1}{8}\left(a_{11}+a_{21}\right) \xi_{1} e^{\xi_{1}}-\frac{1}{8}\left(a_{11}+a_{21}\right) \xi_{1} e^{-\xi_{1}} \\
+\frac{1}{32}\left(a_{11}-a_{13}\right) e^{3 \xi_{1}}+\frac{1}{32}\left(a_{21}-a_{23}\right) e^{-3 \xi_{1}} \\
\Psi_{1 k}\left(\xi_{1}\right)=a_{3 k} e^{k \xi_{1}}+a_{4 k} e^{-k \xi_{1}}+\frac{1}{16(1-k)}\left(a_{1, k}-a_{1, k-2}\right) e^{(k-2) \xi_{1}}+\frac{1}{16(1+k)}\left(a_{1, k}-a_{1, k+2}\right) e^{(k+2) \xi_{1}} \\
+\frac{1}{16(1-k)}\left(a_{2, k}-a_{2, k-2}\right) e^{-(k-2) \xi_{1}}+\frac{1}{16(1+k)}\left(a_{2, k}-a_{2, k+2}\right) e^{-(k+2) \xi_{1}}, \quad k \geq 2 .
\end{gathered}
$$

Formula (9) is a general solution of equation (1). Each function $\Psi_{1 k}\left(\xi_{1}\right)$ depends on the coefficients from sets $a_{1}, a_{2}, a_{3}, a_{4}$. Here, $a_{1}=a_{11} \ldots a_{1(n-2)}, a_{2}=a_{21} \ldots a_{2(n-2)}, a_{3}=a_{31} \ldots a_{3 n}$, $a_{4}=a_{41} \ldots a_{4 n}$. We then need to find coefficients $a_{1}, a_{2}, a_{3}, a_{4}$ to satisfy the boundary conditions for the stream function. Conditions (4),(6) are satisfied for arbitrary values of the coefficients $a_{1}, a_{2}, a_{3}, a_{4}$.

We now denote $\Psi_{1 k}\left(a_{1}, a_{2}, a_{3}, a_{4}\right)$ by $\Psi_{1 k}\left(\xi_{1}\right)$. Condition (5) leads to the algebraic relations between coefficients:

$$
\begin{aligned}
& \left.\Psi_{1 k}\left(a_{1}, a_{2}, a_{3}, a_{4}\right)\right|_{\xi_{1}=\xi_{01}}=0, \\
& \left.\Psi_{1 k}\left(a_{1}, a_{2}, a_{3}, a_{4}\right)\right|_{\xi_{1}=\xi_{02}}=0, \quad k=1 \ldots n .
\end{aligned}
$$

The first condition in (7) takes the form:

$$
\left.\sum_{k=1}^{n} \frac{\partial}{\partial \xi_{1}} \Psi_{1 k} \sin \left(k \xi_{2}\right)\right|_{\xi_{1}=\xi_{01}}=V_{t o p}\left(\xi_{2}\right) d \sqrt{\cosh ^{2}\left(\xi_{01}\right)-\cos ^{2}\left(\xi_{2}\right)} .
$$


Let us consider the function in the right hand side as a Fourier series:

$$
\left.\sum_{k=1}^{n} \frac{\partial}{\partial \xi_{1}} \Psi_{1 k} \sin \left(k \xi_{2}\right)\right|_{\xi_{1}=\xi_{01}}=\sum_{k=1}^{n} C_{k 1} \sin \left(k \xi_{2}\right)
$$

where

$$
C_{k 1}=e^{-k \xi_{01}} \frac{-1}{k \pi} d \int_{0}^{\pi} V_{t o p}\left(\xi_{2}\right) \sqrt{\cosh ^{2}\left(\xi_{01}\right)-\cos ^{2}\left(\xi_{2}\right)} \sin \left(k \xi_{2}\right) d \xi_{2} .
$$

The coefficients $C_{k 1}$ can easily be found numerically. The means of calculating $C_{k 2}$ for the second condition in (7) is analogous. Thus, condition (7) can be represented as an algebraic relation between coefficients:

$$
\begin{gathered}
\left.\frac{\partial}{\partial \xi_{1}} \Psi_{1 k}\left(a_{1 k}, a_{2 k}, a_{3 k}, a_{4 k}\right)\right|_{\xi_{1}=\xi_{01}}=C_{k 1}, \quad k=1 \ldots n \\
\left.\frac{\partial}{\partial \xi_{1}} \Psi_{1 k}\left(a_{1 k}, a_{2 k}, a_{3 k}, a_{4 k}\right)\right|_{\xi_{1}=\xi_{02}}=C_{k 2}, \quad k=1 \ldots n .
\end{gathered}
$$

As a result, coefficients $a_{1}, a_{2}, a_{3}, a_{4}$ are the solutions for a system of linear equations. This system includes the sets of equations (10) and some equations from the sets (12), (13).

\section{Discussion}

The expression for stream function (9) has been completely defined above. If we fix ellipse half axes and vary the velocity of the moving part of the boundary, we can obtain different pictures of the flow. The following figures shows fluid streamlines for different velocity functions at $S_{1}$ and $S_{2}$.

For all the examples described below, the ellipse half axes are $a=0.4 ; b=0.12$ for $S_{1}$ and $a=0.86 ; b=0.76$ for $S_{2}$.
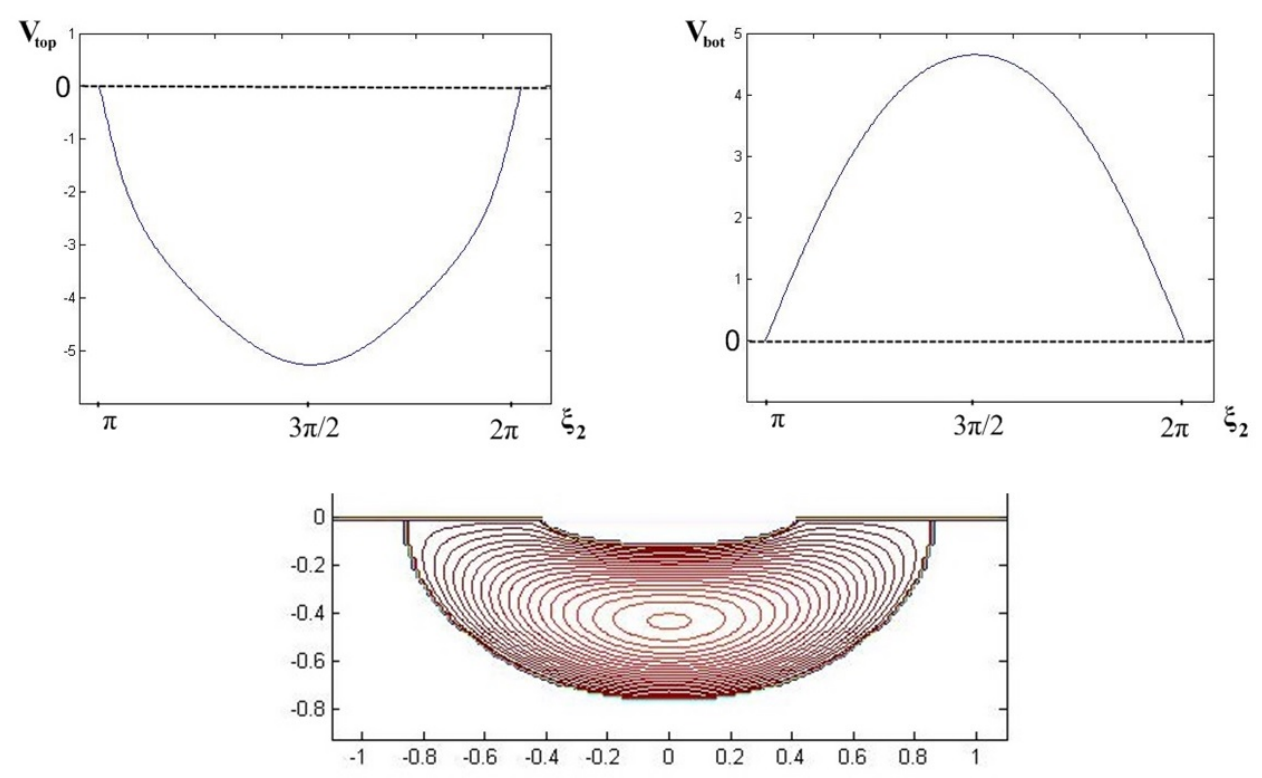

FIG. 3. Velocities on $S_{1}$ and $S_{2}$ have opposite directions. Functions $V_{\text {top }}\left(\xi_{2}\right)$ and $V_{b o t}\left(\xi_{2}\right)$ do not change sign. We have no separation points at the boundary. The whole domain is a single vortex region 

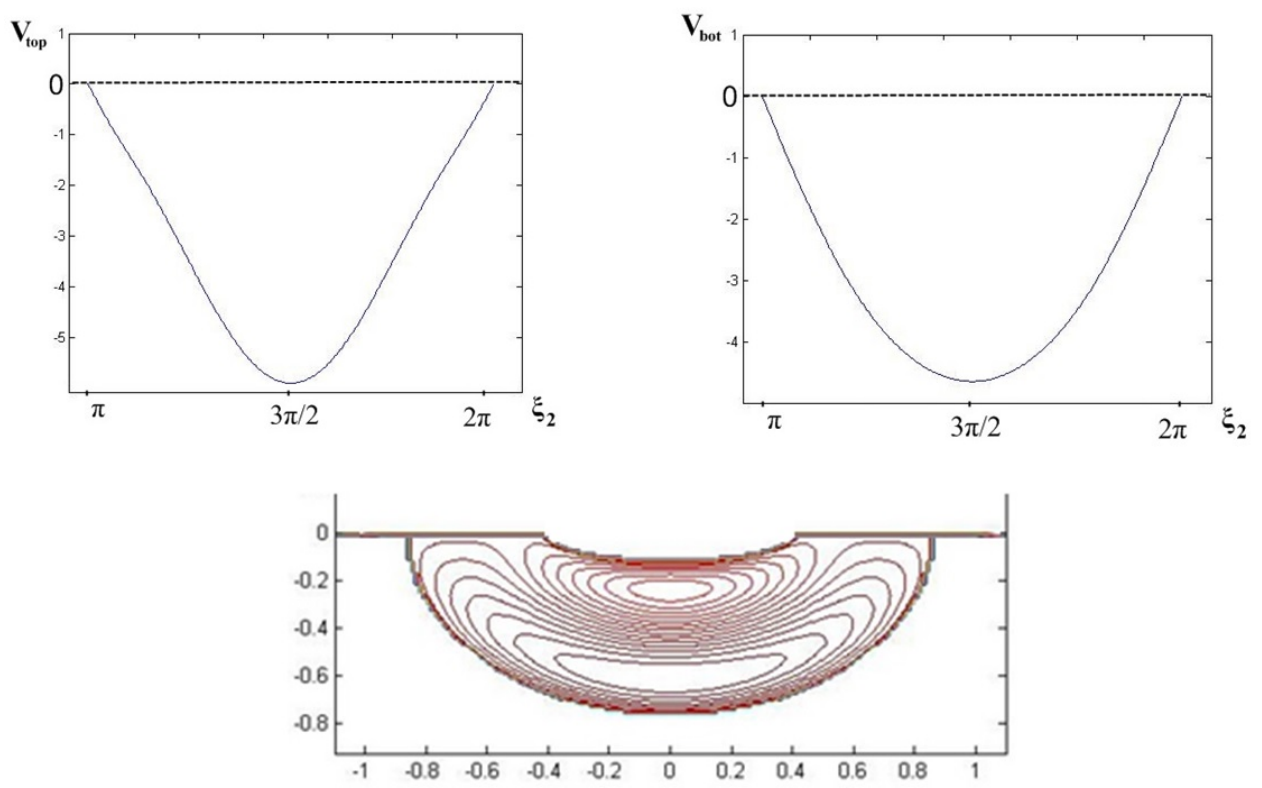

FIG. 4. Functions $V_{S_{1}}\left(\xi_{2}\right)$ and $V_{S_{2}}\left(\xi_{2}\right)$ do not change sign. We have no separation points at $S_{1}$ and $S_{2}$. Velocities on $S_{1}$ and $S_{2}$ are in the same direction. We obtain separation points at segments $A B$ and $C D$. The domain is divided into two subdomains. On the centerline $(x=0)$, we obtain two stagnation points (at these points the fluid is totally stationary), we compare that with [20]
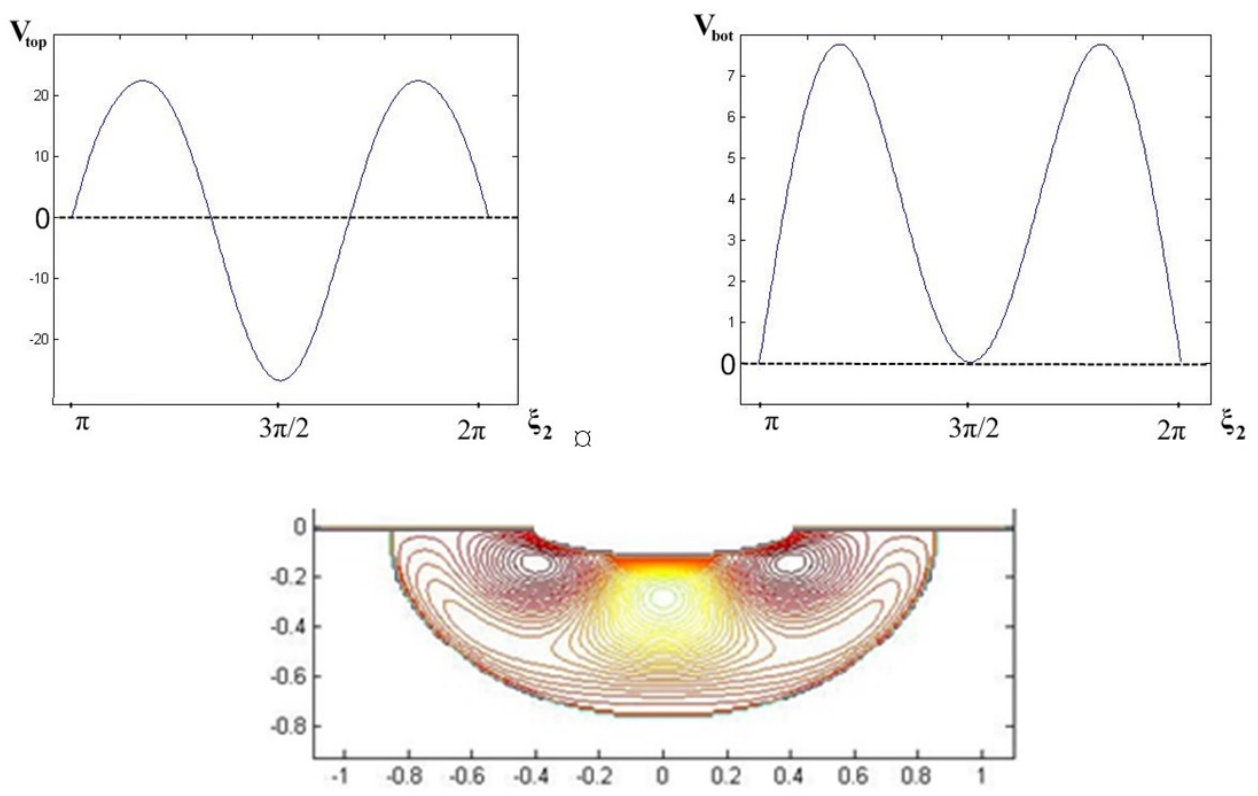

FIG. 5. Function $V_{S_{1}}\left(\xi_{2}\right)$ changes its sign. We obtain two separation points at $S_{1}$. Function $V_{S_{2}}\left(\xi_{2}\right)$ doesn't change its sign. We have no separation points at $S_{2}$. The velocities at the corners of $S_{1}$ and $S_{2}$ are in the same direction. We obtain a single separation point for each segment, $A B$ and $C D$ 


\section{Numerical analysis}

We construct the solution of Eq.(1) by a finite-difference method. The discrete expression for the biharmonic equation, using forward finite difference method, has the form:

$$
\begin{aligned}
& 20 \Psi_{0}-8\left(\Psi_{1}+\Psi_{2}+\Psi_{3}+\Psi_{4}\right)+\Psi_{5}+\Psi_{7}+ \\
& +\Psi_{9}+\Psi_{11}+2\left(\Psi_{6}+\Psi_{8}+\Psi_{10}+\Psi_{12}\right)=0 .
\end{aligned}
$$

The stencil for the finite difference scheme is shown in Fig. 6. The node with number 0 corresponds to a stream function node for which the biharmonic equation is formulated.

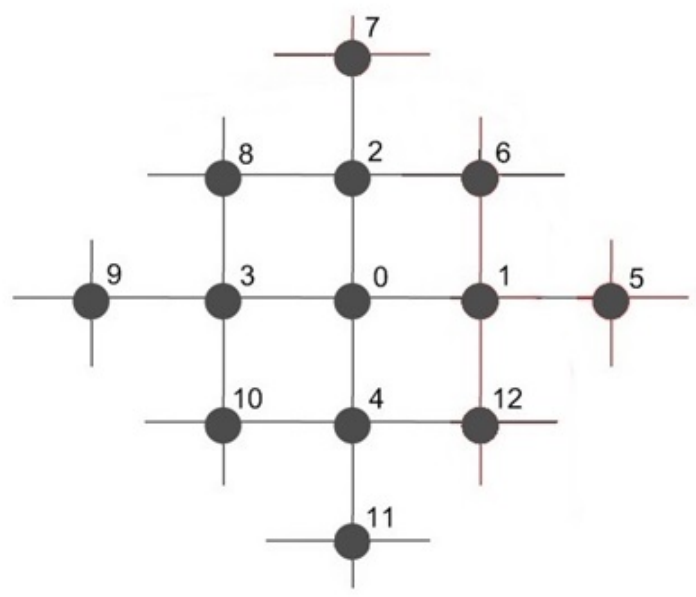

FIG. 6. Stencil nodes numbering

We calculate the relative errors with $L_{1}$ norm and estimate the quality of numerical solution. The dependence of the relative error $E$ via the grid step $d$ for Fig. 4 is shown in Fig. 7. The positive slope confirms the convergence of the algorithm.

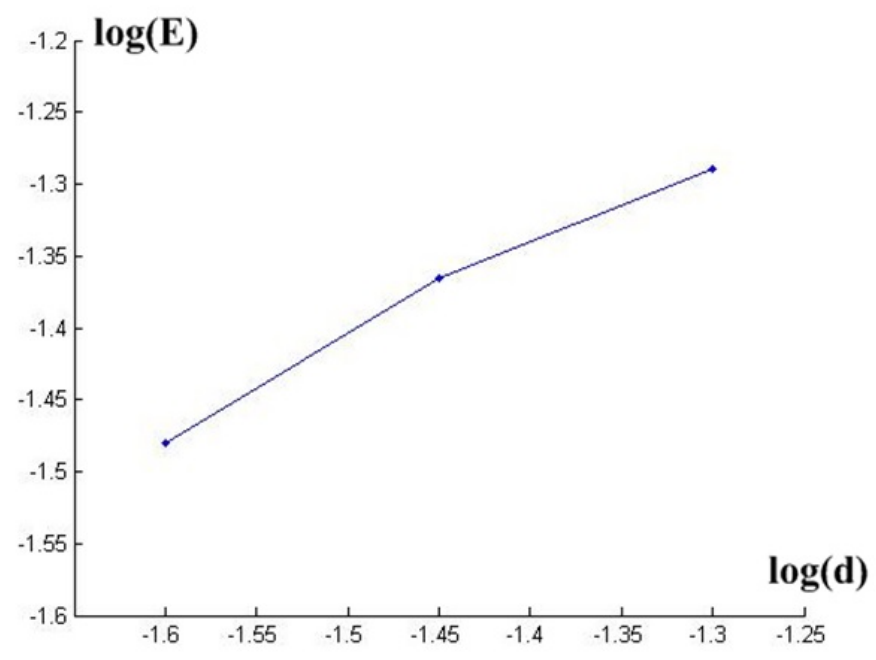

FIG. 7. Error norm via the grid resolution (logarithmic scale) 


\section{Conclusion}

We obtained exact solutions for the biharmonic equation for the stream function of the Stokes flow. We fixed the geometrical parameters of the domain and varied the boundary conditions. Doing so allowed us to change the flow structure drastically. This could open up opportunities for controlling flow structure by external actions.

\section{Acknowledgements}

This work was partially financially supported by the Government of the Russian Federation (grant 074-U01), by Ministry of Science and Education of the Russian Federation (GOSZADANIE 2014/190, Projects No 14.Z50.31.0031 and No. 1.754.2014/K), by grant MK5001.2015.1 of the President of the Russian Federation, by grant 16-11-10330 of Russian Science Foundation.

\section{References}

[1] Li D. Encyclopedia of Microfluidics and Nanofluidics, 2008 Springer, New York.

[2] Rivera J. L. and Starr F. W., Rapid transport of water via carbon nanotube. J. Phys. Chem. C, 2010, 114, P. 3737-3742.

[3] Kang W. and Landman U., Universality crossover of the pinch-off shape profiles of collapsing liquid nanobridges. Phys. Rev. Lett., 2007, 98, P. 064504/1-4.

[4] Chivilikhin S.A., Popov I.Yu., Gusarov V.V., and Svitenkov A.I., Model of fluid flow in a nano-channel. Russian J. Math. Phys., 2007, 15, P. 410-412.

[5] Maslov V.P. Superfluidity of classical liquid in a nanotube for even and odd numbers of neutrons in a molecule. Theor. Math. Phys., 2007, 153, P. 1677-1696.

[6] Belonenko M.B., Chivilikhin S.A., Gusarov V.V., Popov I.Yu., and Rodygina O.A. Soliton-induced flow in carbon nanotube. Europhys. Lett., 2013, 101, P. 66001/1-3.

[7] Popov I.Yu. Statistical derivation of modified hydrodynamic equations for nanotube flows. 2011, Physica Scripta, 83, P. 045601/1-3.

[8] Chivilikhin S.A., Gusarov V.V., Popov I.Yu. Flows in nanostructures: hybrid classical-quantum models. Nanosystems: Phys. Chem. Math., 2012, 3(1), P. 7-26.

[9] Kononova S.V., Korytkova E.N., Romashkova K.A., Kuznetsov Yu.P., Gofman I.V., Svetlichnyi V.M., Gusarov V.V. Nanocomposite on the basis of amide imide resin with hydrosylicate nanoparticles of different morphology. J. Appl. Chem., 2007, 80(12), P. 2064-2070.

[10] Gusarov V.V. and Popov I.Yu. Flows in two-dimensional non-autonomous phases in polycrystalline system. Nuovo Cimento D,1996, 18(7), P. 799-805.

[11] Ismail-Zadeh A. and Tackley P., Computational Methods for Geodynamics, 2010, Cambridge University Press, Cambridge.

[12] Gürcan F. Streamline Topologies in Stokes Flow Within Lid-Driven Cavities. Theoretical and Computational Fluid Dynamics, 2003, 17(1), P. 19-30.

[13] Gürcan F., Gaskell P.H., Savage M.D., and Wilson M.C.T. Eddy genesis and transformation of Stokes flow in a double-lid driven cavity. J. of Mechanical Engineering Science, 2003, 217,P. 353-363.

[14] Shankar P.N. The eddy structure in Stokes flow in a cavity. J. Fluid Mechanics, 1993, 250, P. 371-383.

[15] Meleshko V.V. Steady Stokes flow in a rectangular cavity. Proc. R. Soc. Lond, 1996, A. 452, P. 1999-2022.

[16] van der Woude D., Clercx H.J.H., van Heijst G.J.F., and Meleshko V.V. Stokes flow in a rectangular cavity by rotlet forcing. Phys. Fluids, 2007, 19(8), P. 083602.

[17] Popov I.Yu. Operator extensions theory and eddies in creeping flow. Phys. Scr., 1993, 47, P. 682-686.

[18] Popov I.Yu. Stokeslet and the operator extensions theory. Rev. Mat. Univ., Compl. Madrid, 1996, 9(1), P. $235-258$.

[19] Sturges L. D. Stokes flow in a two-dimensional cavity with moving end walls. Phys. Fluids, 1986, 29(5), P. 1731-1734.

[20] Krasnopolskaya N.S., Meleshko V.V., Peters G.W.M., Meijer H.E.Y., Steady Stokes flow in an annular cavity, Q. J. Mech. Appl. Math., 1996, 49(4), P. 593-619.

[21] Hackborn W. W. An analysis of a Stokes flow in an annular region. Canadian Applied Mathematics Quarterly, 2000, 8, P. 171-183. 
[22] Murad A, Sen. Stokes Flow between Two Cylinders. Journal of Bangladesh Academy of Sciences, 2012, 36(1), P. 123-135.

[23] Gaskell P.H., Savage M.D., and Wilson M. Stokes flow in a half-filled annulus between rotating coaxial cylinders. J. Fluid Mechanics, 1997, 337, P. 263-282.

[24] Blinova I.V. Model of non-axisymmetric flow in nanotube. Nanosystems: Phys. Chem. Math., 2013, 4(3), P. 320-323.

[25] Gugel Yu.V., Popov I.Yu., and Popova S.L. Hydrotron: creep and slip. Fluid Dyn. Res., 1996, 18, P. $199-210$.

[26] Saatdjian E., Midoux N., and André. On the solution of Stokes' equations between confocal ellipses. Phys. Fluids, 1994, 6, P. 3833.

[27] Popov I.Yu., and Makeev I.V. A benchmark solution for 2-D Stokes flow over cavity. Z. Angew. Math. Phys., 2014, 65, P. 339-348.

[28] Moffatt H.K. Viscous and resistive eddies near a sharp corner, J. Fluid Mech., 1964, 18, P. 1-18.

[29] Moffatt H.K. Viscous eddies near a sharp corner. Arch. Mech. Stosowanej, 1964, 16, P. 365-372.

[30] Blinova I.V., Kyz'yurova K.N., and Popov I.Yu. Stokes flow driven by a Stokeslet in a cone. Acta Mechanica, 2014, 225, P. 3115-3121.

[31] Gerya T. Introduction to Numerical Geodynamic Modelling, 2010, Cambridge University Press, Cambridge. 